\title{
Noise Removal in Image Using LPG-PCA (Local Pixel Grouping- Principle Component Analysis) Algorithm
}

\author{
G.Hari Priya ${ }^{1,}$ B.Venkatesh ${ }^{2}$, A.Srivani ${ }^{3}$, G.Sowmya ${ }^{4}$ N.Rajasekhar ${ }^{5}$ \\ ${ }^{1-4}$ (Pursing B.Tech, Department of Electronics \& Communication Engineering, lendi institute of Engineering \\ and technology/JNTUK, India) \\ ${ }_{5}^{5}$ (Assistant Professor Department of Electronics \& Communication Engineering, lendi institute of Engineering \\ and technology/JNTUK, India)
}

\begin{abstract}
In this paper an effective algorithm for noise removal in an image is obtained by using PCA (principal component analysis) with LPG (Local Pixel Grouping). This technique ensures the preservation of image local structure. Here the pixels and its neighbors are treated as vector variables whose training samples are selected from local windows using block matching based LPG. This ensures only the similar samples are selected for the PCA transformation so that the desired local characteristics are only preserved with considerable noise reduction. The LPG-PCA algorithm is performed twice to enhance the quality of an image. The first iteration would remove the noise considerably and the second iteration would preserve the image features like edges etc. The LPG-PCA algorithm will adaptively adjust the noise level of an image unlike WT (Wavelet Transformation). Several experimental results show the effectiveness of the proposed algorithm.
\end{abstract}

Keywords: PCA (Principal Component Analysis), LPG (Local Pixel Grouping)

\section{Introduction:}

During the process of image acquisition every image is prone to certain amount of noise. Noise removal is the primary step in order to improvise the image quality. As noise removal appears to be the key hindrance in achieving the desired image it is extensively studied and several noise removal algorithms have been proposed so far. Several noise removal techniques [17]starting from smoothening filters, frequency domain filtering techniques and recently developed techniques like wavelets [5], curvlets [6] and ridgelets [7] have been proposed to remove noise. Some more techniques like sparse representation [8], K-SVD Methods [9], shapeadaptive transform [10], bilateral filtering [11,12], and non-local mean based methods [13,14] and non-localcollaborative filtering [15].

Initial smoothening filter techniques could not offer the desired results, Wavelets Transforms [10] are able to effectively reduce the image noise .In this technique the input image is divided into multiple scales which are uniquely transformed into their frequency domain. Then process like thresholding is applied on them. Noise removal is attained by inverse transforming the image into its spatial domain. The WT though effective in noise removal is non adaptive hence it does not yield desired results on the real time images. The wavelets technique is advanced as curvlets and ridgelets.

To overcome the disadvantage in WT [21] an adaptive statistical approach called PCA (principal component Analysis was developed by Muresan and Parks.Elad and Ehersonproposed the sparse representation method and K-SVD method [14]. The recently developed NLM (Non Local Means) method is used to effectively remove the noise it is different from other conventional methods because of its unique approach. The NLM [21] uses the pixel averages based on the intensity distances.

In this paper we present LPG-PCA technique for noise removal in an image. PCA is a de-correlation technique in statistical signal processing used pervasively in pattern recognition. By transforming th image data set into PCA domain and preserving only the desired components the noise and other trivial information can be removed considerably. In the proposed LPG-PCA algorithm the input dataset to PCA is obtained using the block match LPG technique. Here the pixels and its neighbors are modeled as vectors and the training samples are determined by selecting the pixels with similar properties within the local window. This algorithm ensures effective noise removal and edge preservation. The algorithm is computed in two stages for effectiveness.

In this paper section 2 deals with PCA, section 3 deals with LPG-PCA algorithm in detail, section-4 deals with results of proposed algorithm and section-5 includes conclusions.

II. PCA (principle component analysis):

Let $\mathrm{X}=\left[\mathrm{x}_{1}, \mathrm{x}_{2}, \mathrm{x}_{3} \ldots \ldots \mathrm{x}_{\mathrm{m}}\right]^{\mathrm{T}}$ denote an $\mathrm{m}$ component vector in its transpose form. This is denoted as 


$$
X=\left[\begin{array}{ccccc}
x_{1}^{1} & x_{1}^{2} & \ldots \ldots \ldots & x_{1}^{n} \\
x_{2}^{1} & x_{2}^{2} \ldots \ldots \ldots & x_{2}^{n} \\
\vdots & & & \\
\vdots & & & \\
x_{m}^{1} & x_{m}^{2} & \ldots \ldots \ldots & x_{m}^{n}
\end{array}\right]
$$

The sample matrix of $X$ where $\mathrm{Xij}$ where $\mathrm{j}=1,2 \ldots \mathrm{nT}$ represent the disctere sample variables of the sample vector $\mathrm{Xi}$ where $\mathrm{i}=1,2,3 \ldots \mathrm{m}$.

The mean value of sample vector is calculated as $\mu_{i}=\frac{1}{n} \sum_{j=1}^{n} X_{i}(j)$

Thus the average value of the pixel is computed using the above equation The sample vector is modified and centralized as follows

$\bar{X}_{i}=X_{i}-\mu_{i}=\left[\begin{array}{lll}\bar{x}_{i}^{1} & \bar{x}_{i}^{2} \ldots \ldots \bar{x}_{i}^{n}\end{array}\right]$

Finally we calculate the covariance matrix using the formula

$\Omega=\frac{1}{n} \overline{X X}^{T}$

The goal of PCA is to generate an orthogonal transformation matrix $\mathrm{P}$ to de-correlate the original matrix such that $\mathrm{Y}=\mathrm{PX}$, such that the covariance matrix of $\mathrm{Y}$ is diagonal. Since the covariance matrix is symmetrical it can be written as

$\Omega=\Phi_{\Lambda} \Phi^{T}$

Where $\Omega$ is $\mathrm{m} \times \mathrm{m}$ eigenvector matrix and $\wedge$ is a diagonal eigenvector matrix. Based on the eigen values the orthogonal transformation matrix $\mathrm{P}$ is given as $\mathrm{P}=\Phi^{T}$

Thus the matrix $\mathrm{X}$ can be de-correlated using the orthogonal transformation matrix $\mathrm{P}$ as $\mathrm{Y}=\mathrm{PX}$.

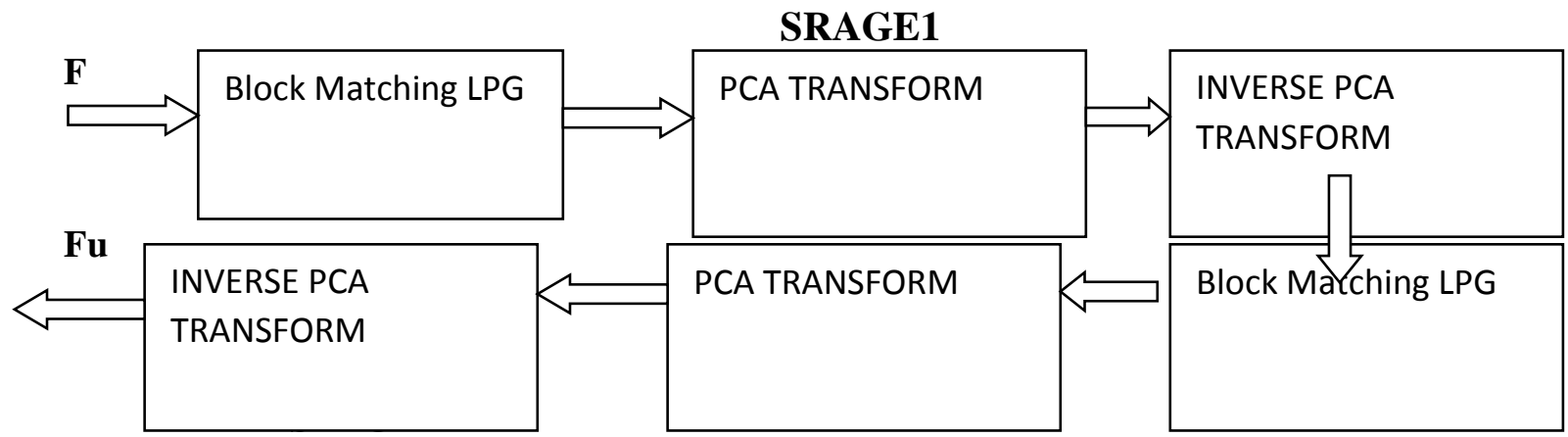

Fig: 2-Stage implementation of LPG-PCA Algorithm

III. LPG-PCA algorithm:

Here we assume that the noise (u) in the image is additive, with zero mean and standard deviation $\sigma$. Let this noise be added to the original image say $F$. Therefore the new image value is determined as $F_{u}=F+u$. The goal of our project is to find an image $\mathrm{F}^{1}$ which is approximately equal to the original image F. Pixels are identified based on the spatial coordinates and their grey scale value( intensity value) whereas of different intensity values. Here we assume the pixels in local structure as vectors and improvise the edge preservation process. The image $\mathrm{F}$ and noise $\mathrm{u}$ are uncorrelated. For removing noise from an underlying pixel, according to the fig, a $\mathrm{K} \times \mathrm{K}$ matrix centered on the pixel and denote by $\mathrm{X}=\left[\mathrm{x}_{1}, \mathrm{X}_{2} \ldots \mathrm{x}_{\mathrm{m}}\right]^{\mathrm{T}}$ with total no of elements $\mathrm{m}=\mathrm{k}^{2}$. The window is centered on the image $X$. Since the image is prone to noise $u$ we represent the new image vector as $X_{u}=X+u$. The noisy image where $U=\left[u_{1}, u_{2} \ldots u_{m}\right]^{T}$. The statistical PCA is used on these vectors. To remove the noise from an image the covariance matrix $X_{u}$ and PCA transformation matrix are to be calculated. Therefore, we use a LL training block centered on $X_{u}$, such that $L \times L$ is greater than $K \times K$. From the training block we need to estimate the required pixels for the PCA. This selection of different pixels from training blocks is a complex process and may sometimes leads to inaccurate results. 


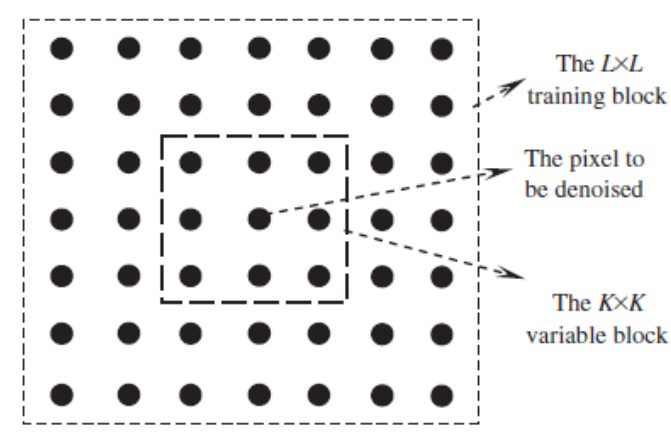

Fig. 2. Illustration of the modeling of LPG-PCA based denoising.

\subsection{LPG Technique:}

Here selecting the training samples similar to the $\mathrm{K} \times \mathrm{K}$ central block from the given $\mathrm{L} \times \mathrm{L}$ training block is achieved using block matching based technique. The total number of samples available are $(\mathrm{L}-\mathrm{K}+1)^{2}$ training samples of $\mathrm{Xu}$ in the $\mathrm{L} \times \mathrm{L}$ training window. In the $\mathrm{L} \times \mathrm{L}$ training block, let $\mathrm{x}_{0}$ denote the vector containing sample pixels in the center $\mathrm{K} \times \mathrm{K}$ block and $\mathrm{x}_{\mathrm{i}}$ represent the pixels of $(\mathrm{L}-\mathrm{K}+1)^{2}-\mathrm{I}$.

\subsection{LPG-PCA based denoising:}

In the $\mathrm{m} \times \mathrm{n}$ dataset matrix $X_{u}$, eachcomponent $x_{k}^{u}, \mathrm{k}=1,2, \ldots \mathrm{m}$, of the vector variable $x_{u}$ has $\mathrm{n}$ samples. Denote by $x_{k}^{u}$ the row vector containing the $\mathrm{n}$ samples of $x_{k}^{u}$. The $\mathrm{n}$ the dataset $x_{u}$ can be represented as $X_{u}=\left[\left(X_{1}^{u}\right)^{T} \ldots \ldots\left(X_{m}^{u}\right)^{T}\right]^{T}$. Similarly, we have $\mathrm{X}=\left[X_{1}^{T} \ldots \ldots \ldots \ldots X_{m}^{T}\right]^{T}$, where $X_{k}$ is the row vector containing the n samples of $x_{k}$, and $\quad X_{u}=\mathrm{X}+\mathrm{V}$,

Where $\mathrm{V}=\left[\begin{array}{llll}V_{1}^{T} & \ldots \ldots \ldots & \ldots & \ldots \\ m\end{array}\right]^{T}$ is the dataset of noise variable $\mathrm{u}$ and $V_{k}$ is sample vector $u_{k}$.

Next we centralized a set $X_{u}$. Themeanvalueof $X_{k}^{u}$ is $\mu_{k}=\frac{1}{n} \sum_{i=1}^{n} X_{k}^{u}(i)$, andthen $X_{k}^{u}$ is centralized by $\bar{X}_{k}^{u}=X_{k}^{u}-$ $\mu_{k}$. Since the noise $u_{k}$ is zero-mean, $X_{k}$ can also be centralized by $\bar{X}_{k}=X_{k}-\mu_{k}$. Then centralized datasets of $X_{u}$ and $X$ are obtainedas $\bar{X}_{u}=\left[\left(\bar{X}_{1}^{u}\right)^{T} \ldots \ldots\left(\bar{X}_{m}^{u}\right)^{T}\right]^{T}$ and $\bar{X}=\left[\bar{X}_{1}^{T} \ldots \ldots \ldots \ldots \bar{X}_{m}^{T}\right]^{T}$, andwehave $\bar{X}_{u}=\bar{X}+\mathrm{V}$.

By computing the covariance matrix of $\bar{X}$, denotedby $\Omega_{\bar{x}}$, thePCA transformation matrix $P_{\bar{x}}$ can be obtained.

$$
\Omega_{\overline{x_{u}}}=\frac{1}{n} \bar{X}_{u} \bar{X}_{u}^{T} \approx \frac{1}{n}\left(\overline{X X^{T}}+\bar{X} V^{T}+V \bar{X}^{T}+V V^{T}\right)
$$

Since $\bar{X}$ and $\mathrm{V}$ are uncorrelated, items $\bar{X} V^{T}$ and $V \bar{X}^{T}$ will benearly zero matricesandthus:

$\Omega_{\overline{x_{u}}} \approx \frac{1}{n}\left(\overline{X X^{T}}+V V^{T}\right)=\Omega_{\bar{x}}+\Omega_{u}$

Where $\Omega_{\bar{x}}=(1 / \mathrm{n}) \overline{X X}^{T}$ and $\Omega_{u}=(1 / \mathrm{n}) V V^{T}$.

The component $\Omega_{u}(i, j)$ is the correlation between $u_{i}$ and $u_{j}$. Since $u_{i}$ and $u_{j}$ are un co-related for $\mathrm{i} \neq \mathrm{j}$, we know that $\Omega_{u}$ isa $m \times m$ diagonal matrix with all the diagonal components being $\sigma^{2}$. In other words, $\Omega_{u}$ can be written as $\sigma^{2} \mathrm{I}$, where I is theidentity matrix. Then it can be readily proved that the PCA transformation matrix $P_{\bar{x}}$ associatedwith $\Omega_{\bar{x}}$ is same asPCA transformation matrix associated with $\Omega_{\overline{x_{u}}}$.

Since $\Omega_{\bar{x}}$ is written as

$$
\Omega_{\bar{x}}=\Phi_{\bar{x} \wedge_{\bar{x}}} \Phi_{\bar{x}}^{T}
$$

Where $\Phi_{\bar{x}}$ is the $m \times m \iota_{\bar{x}}$ is the diagonal eigen value matrix. Since $\Phi_{\bar{x}}$ is an orthonormal matrix, we can write $\Omega_{u}$ as

$\Omega_{u}=\left(\sigma^{2} \mathrm{I}\right) \Phi_{\bar{x}} \Phi_{\bar{x}}{ }^{T}=\Phi_{\bar{x}}\left(\sigma^{2} \mathrm{I}\right) \Phi_{\bar{x}}{ }^{T}=\Phi_{\bar{x}} \Omega_{u} \Phi_{\bar{x}}{ }^{T}$

Thus we have

$\Omega_{\overline{x_{u}}}=\Omega_{\bar{x}}+\Omega_{u}=\Phi_{\bar{x}} \Lambda_{\bar{x}} \Phi_{\bar{x}}^{T}+\Phi_{\bar{x}}\left(\sigma^{2} \mathrm{I}\right) \Phi_{\bar{x}}{ }^{T}$

$=\Phi_{\bar{x}}\left(\Lambda_{\bar{x}}+\sigma^{2} \mathrm{I}\right) \Phi_{\bar{x}}{ }^{T}=\Phi_{\bar{x}}\left(\Lambda_{\bar{x}}\right) \Phi_{\bar{x}}{ }^{T}$

Where $\overline{x_{u}}=\Lambda_{\bar{x}}+\sigma^{2}$ I.above equation implies that $\Omega_{\overline{x_{u}}}$ and $\Omega_{\bar{x}}$ have the same eigen vector matrix $\Phi_{\bar{x}}$. Thus, impractical implementation we can directly compute $\Phi_{\bar{x}}$ by decomposing $\Omega_{\overline{x_{u}}}$, instead of $\Omega_{\bar{x}}$, and thentheorthonormalPCAtransformationmatrixfor $\bar{X}$ is set as

Applying $P_{\bar{x}}$ to dataset $\bar{X}_{u}$, we have

$$
P_{\bar{x}}=\Phi_{\bar{x}}^{T}
$$

$\bar{Y}_{u}=P_{\bar{x}} \bar{X}_{u}=P_{\bar{x}} \bar{X}+P_{\bar{x}} \mathrm{~V}=\bar{Y}+V_{y}$

Where $\bar{Y}=P_{\bar{x}} \bar{X}$ is the de-correlated dataset for $\bar{X}$ and $V_{y}=P_{\bar{x}} \mathrm{~V}$ is the transformed noise dataset for V. Since $\bar{Y}$ and $V_{y}$ noise are uncorrelated, we can easily derive that the covariance matrix of $\bar{Y}_{u}$ is 


$$
\Omega_{\overline{y_{u}}}=\frac{1}{n} \bar{Y}_{u} \bar{Y}_{u}^{T}=\Omega_{\bar{y}}+\Omega_{u_{y}}
$$

Where $\Omega_{\bar{y}}=\Lambda_{\bar{x}}$ is the co variance matrix of de-correlated dataset $\bar{Y}$ and $\Omega_{u_{y}}=P_{\bar{x}} \Omega_{u} P_{\bar{x}}{ }^{T}$ is the covariance matrix of noise dataset $V_{y}$.

In the PCA transformed domain $\bar{Y}_{u}$, most energy of noiseless dataset $\bar{Y}$ concentrate on the most important components, while the energy of noise $V_{y}$ distributesmuchmoreevenly. The noisein $\bar{Y}_{u}$ can be suppressed by using the linear minimum mean square-error estimation (LMMSE) technique .Since $\bar{Y}_{u}$ is centralized ,the LMMSE of $\overrightarrow{\bar{Y}}_{k}$, i.e.the kth rowof $\bar{Y}$, isobtainedas

Where the shrinkage coefficient

$$
\dot{\overline{\bar{Y}}}_{k}=w_{k} \cdot \overline{\bar{Y}}_{u}^{k}
$$

$$
w_{k}=\Omega_{\bar{y}}(k, k) /\left(\Omega_{\bar{y}}(k, k)+\Omega_{u_{y}}(k, k)\right)
$$

and $\overline{\bar{Y}}_{u}^{k}$ is the kth rowof $\bar{Y}_{u}$. Inflatzones, $\Omega_{\bar{y}}(k, k)$ is muchsmaller than $\Omega_{u_{y}}(k, k)$ so that $w_{k}$ is closeto0.Hencemostofthenoisewill be suppressedin $\dot{\overline{\bar{Y}}}_{k}$ byLMMSEoperator $\dot{\bar{Y}}_{k}=w_{k} \cdot \overrightarrow{\bar{Y}}_{u}^{k}$

.In implementation we first calculate $\Omega_{\overline{y_{u}}}$ from the available noisy dataset $\bar{Y}_{u}$ and thenestimate $\Omega_{\bar{y}}(k, k)$ by $\Omega_{\bar{y}}(k, k)=\Omega_{\overline{y_{u}}}(k, k)-\Omega_{u_{y}}(k, k)$. In flatzones, it is often that $\Omega_{\overline{y_{u}}}(k, k)-\Omega_{u_{y}}(k, k) \leq 0$, and then weset $\Omega_{\bar{y}}(k, k)=0$. Inthiscase $w_{k}$ will beexactly0andall the noisein $\overline{\bar{Y}}_{u}^{k}$ will beremoved.

Denoted by $\bar{Y}$ the matrix of all $\dot{\bar{Y}}_{k}$. Bytransforming $\bar{Y}$ back to the time domain, we obtain the de-noised result of $\bar{X}_{u}$ as $\bar{X}=\bar{Y} \cdot P_{\bar{x}}$

We used the fact that $P_{\bar{x}}{ }^{-1}=P_{\bar{x}}{ }^{T}$. Adding the mean values $\mu_{k}$ back to $\widehat{X}$ gives the de-noised dataset $\hat{X}$. The estimation of the central block $\vec{x}_{u}$, denotedas $\vec{x}_{u}$, can then be extracted from $\widehat{X}$ and finally the de-noised result of the underlying central pixel can be extracted from $\vec{x}_{u}$. Applying the above procedure to each pixel leads to the full de-noised image of $I_{u}$.

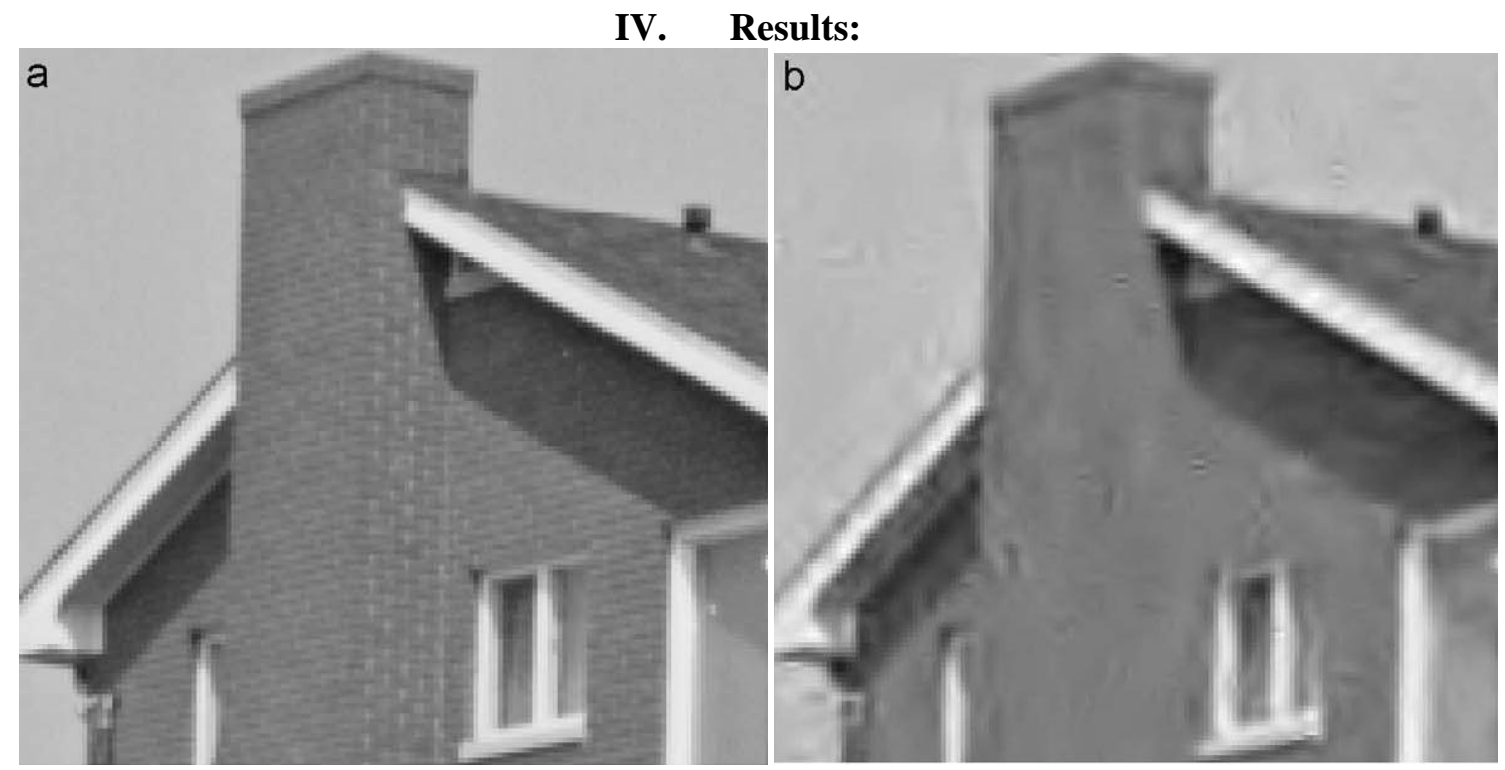

Fig(a) represent-ts original image

fig(b) represents the noisy image 


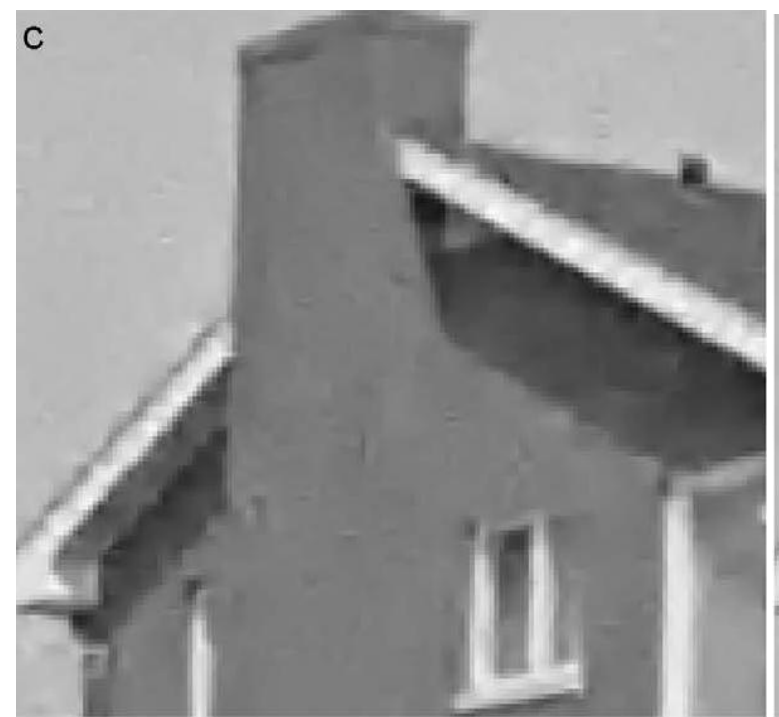

Fig(c) represents The LPG-PCA first stage output

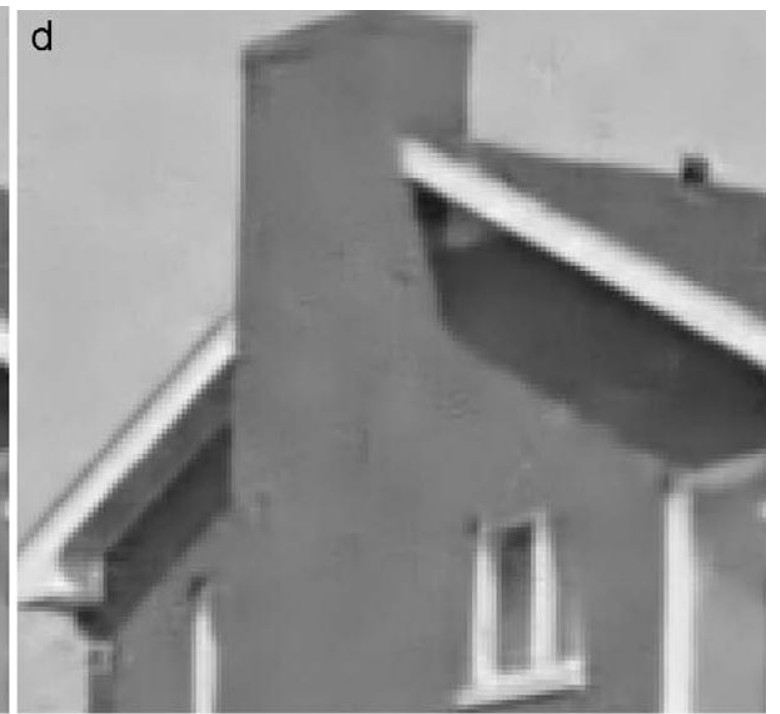

Fig(d) represents LPG-PCA second stage output

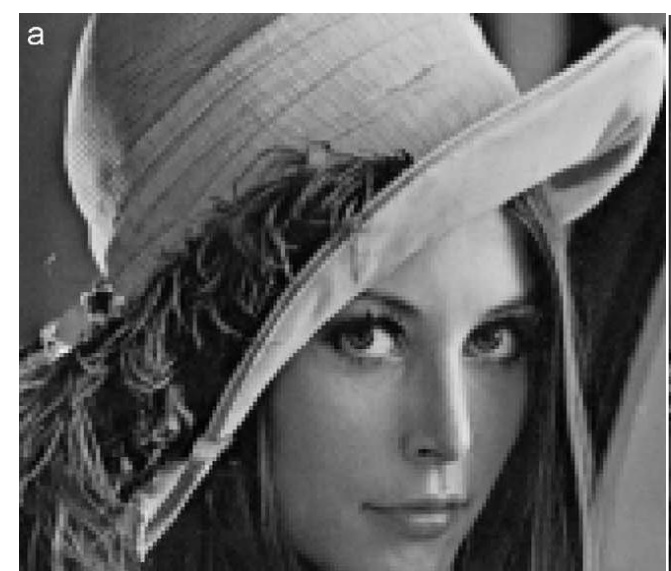

Fig(a) represent-ts original image

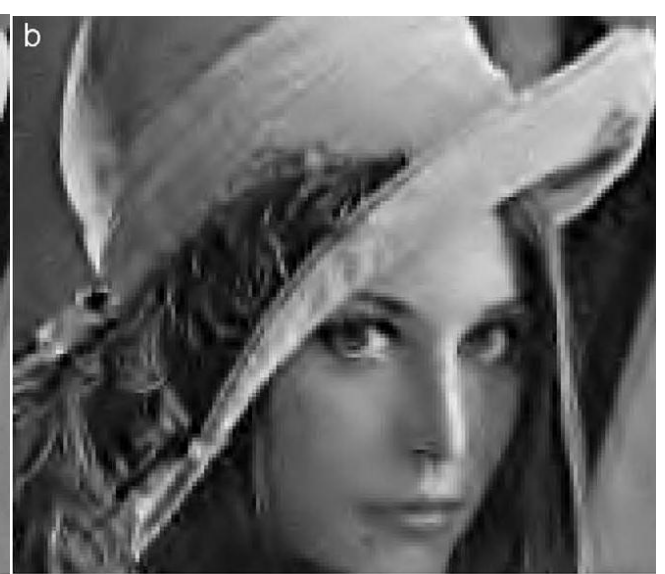

fig(b) represents the noisy image

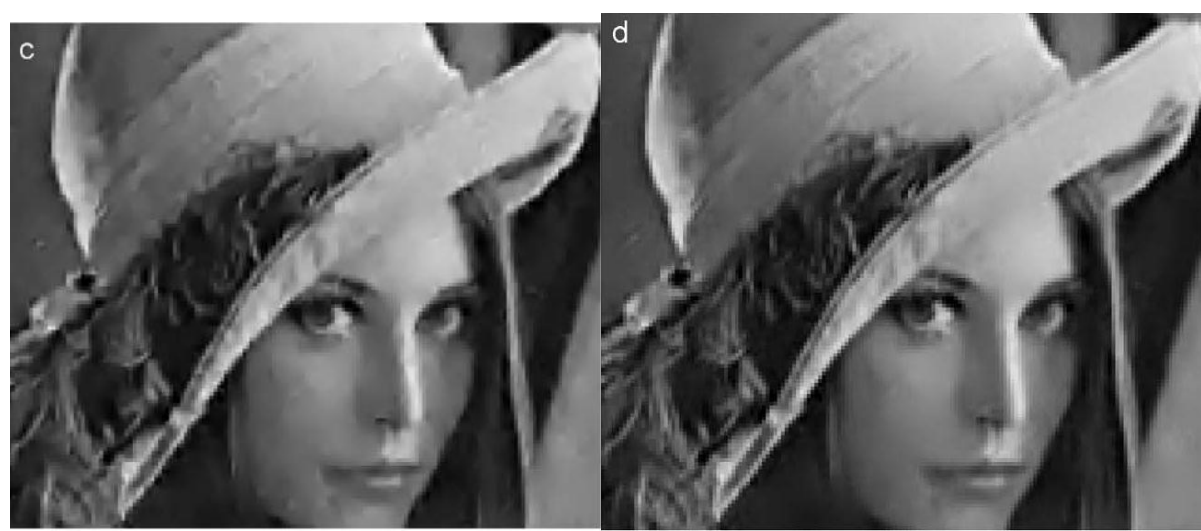

Fig(c) represents The LPG-PCA first stage output

Fig(d) represents LPG-PCA second stage output ,. 

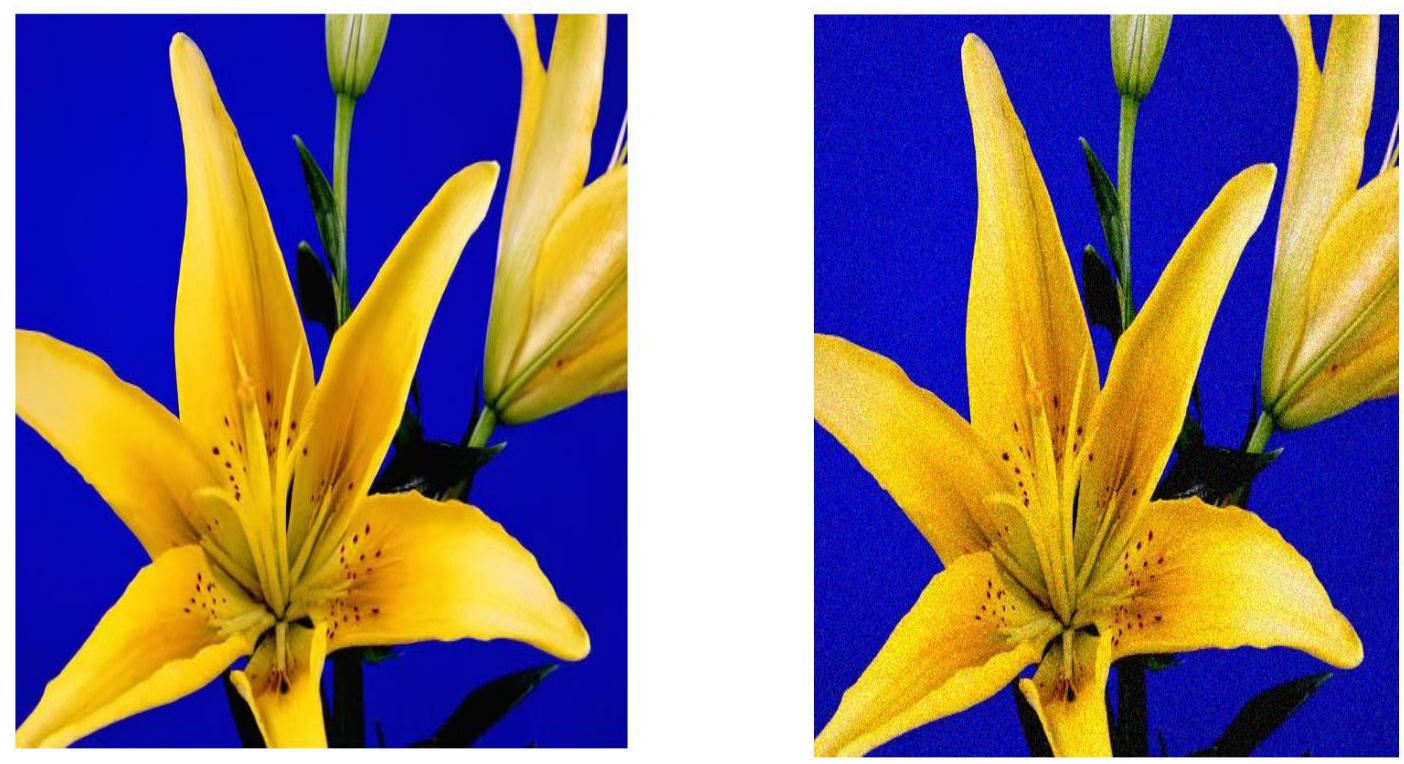

Fig(a) Original Image

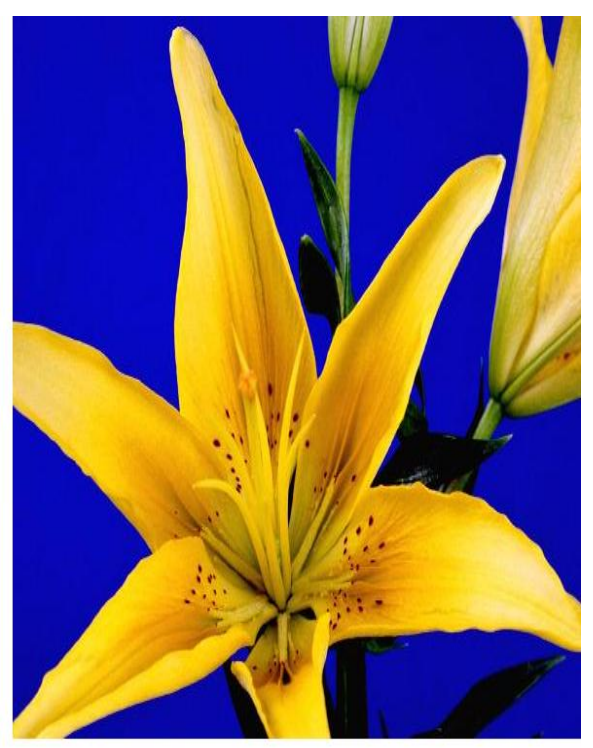

Fig(c ) Stage 1 LPG-PCA output
Fig(b) Noisy Image

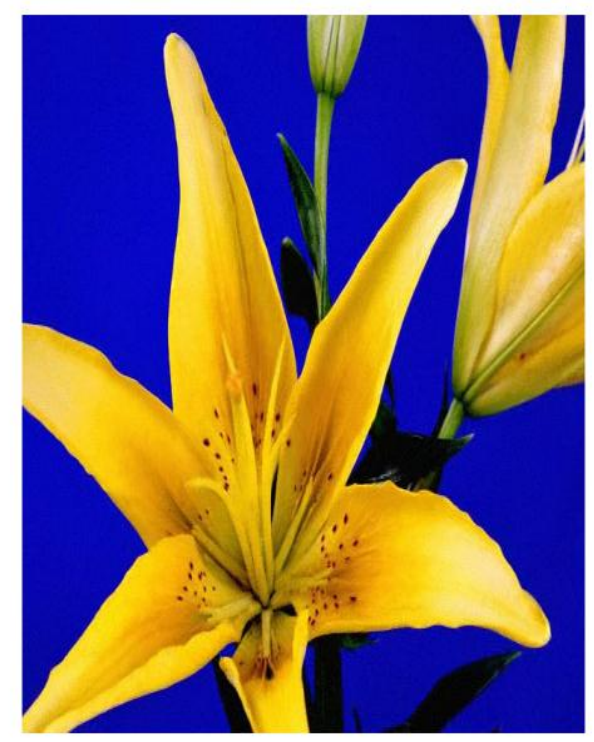

Fig(d) Stage 2 LPG-PCA output 


\begin{tabular}{|l|l|l|l|l|l|l|}
\hline IMAGE & Psnr1 & Psnr2 & Ssim1 & Ssim2 & $\begin{array}{l}\text { Stge(3) } \\
\text { Psnr }\end{array}$ & Stage(3)ssim \\
\hline Image1(House) & 32.224 & 33.082 & 0.809 & 0.867 & 33.1 & 0.87 \\
\hline Image2(Lena) & 30.77 & 32.46 & 0.7887 & 0.8777 & 32.47 & 0.89 \\
\hline Image3(yellow) & 31.661 & 33.576 & 0.7737 & 0.888 & 33.59 & 0.888 \\
\hline
\end{tabular}

The Psnr1 and Psnr2 (Peak signal to noise ratio) for stage 1 and stage 2 are determined, that is fig( c) and fig(d). The results show that the peak signal to noise ratio of stage 2 ie fig (d) yields better results than the stage 1 .

Stage 3 is achieved by applying the LPG-PCA algorithm for the third time.

\section{Conclusions:}

- The paper deals with LPG-PCA algorithm which effectively removes the noise encountered in an image. Here we model the pixels as vector variables.

- Training samples for PCA technique are chosen based on block matching LPG technique.

- The block matching technique ensures that only required pixels are selected for PCA Transformation.

- The selected pixels then undergo PCA and their coefficients are shrunk to obtain the desired results.

- The de-noising technique adopted is an adaptive one and efficiently removes the noise when compared to WT (Wavelet Transformation).

- The proposed LPG-PCA algorithm is iterated one more time for better image characteristic preservation.

- The above experimental results assures the effectiveness of the algorithm.

\section{References:}

[1] D.L Donoho, de-noising by soft thresholding,IEEE Transactions on information theory 41(1995) 613-627.

[2] R.R Coifman, D.L Donoho, Translation-invariant-de-noising,sprinjer,Berlin Journal 1995

[3] M.K Michhack,I.Kozenstev,K.Ramachandran,P.Moulin Low complexity image de-noising based on statistical modelling of wavelet co-efficient, IEEE signal processing Letters 6(12) 1999,300-303.

[4] S.G Chang,B.Yu.M.Vetterli spatially adaptive wavelet thresholding with context modelling for image de-noising, IEEE transaction on image processing 9(9)2000,1522-1531

[5] A. Pizurica,W. Philips, Estimating the probability of the presence of a signal of interest in multi resolution single- and multiband image denoising, IEEE Transaction on Image Processing 15 (3) (2006) 654-665.

[6] J.L. Starck, E.J. Candes, D.L. Donoho, The curvelet transform for image denoising, IEEE Transaction on Image Processing 11 (6) (2002) 670-684.

[7] G.Y. Chen, B. Ke` gl, Image de-noising with complex ridgelets, Pattern Recognition 40 (2) (2007) 578-585

[8] M. Elad-, M. Aharon, Image de-noising via sparse and redundant representation over learned dictionaries, IEEE Transaction on Image Processing 15 (12) (2006) 3736-3745.

[9] M. Aharon, M. Elad, A.M. Bruckstein, The K-SVD: an algorithm for designing of over complete dictionaries for sparse representation, IEEE Transaction on Signal Processing 54 (11) (2006) 4311-4322.

[10] A. Foi, V. Katkovnik, K. Egiazarian, Point wise shape-adaptive DCT for high- quality de-noising and de-blocking of grayscale and color images, IEEE Transaction on Image Processing 16 (5) (2007).

[11] C. Tomasi, R. Manduchi, Bilateral filtering for gray and color images, in: Proceedings of the 1998 IEEE International Conference on Computer Vision, Bombay, India, 1998, pp. 839-846. [12] D. Brash, A fundamental relationship between bilateral filtering, adaptive smoothing, and the nonlinear diffusion equation, IEEE Transaction on Pattern Analysis and Machine Intelligence 24 (6) (2002) 844-847.

[13] A. Buades, B. Co, J.M. Morel, A review of image de-noising algorithms, with a new one, Multiscale Modeling Simulation 4 (2) (2005) 490-530.

[14] C. Kervrann, J. Boulanger, Optimal spatial adaptation for patch based image de-noising, IEEE Transaction on Image Processing 15 (10) (2006) 2866-2878.

[15] K. Dabov, A. Foi, V. Katkovnik, K. Egiazarian, Image de-noising by sparse 3D transform-domain collaborative filtering, IEEE Transaction on Image Proces- sing 16 (8) (2007) 2080-2095.

[16] D.D. Muresan, T.W. Parks, Adaptive principal components and image denoising, in: Proceedings of the 2003 International Conference on Image Processing, 14-17 September, vol. 1, 2003, pp. I101-I10

[17] R.C. Gonzalez, R.E. Woods, Digital Image Processing, second ed., Prentice- Hall, Englewood Cliffs, NJ, 2002 